Relations industrielles

Industrial Relations

\title{
The Social Psychology of Bargaining and Negotiation, by Jeffrey Z. Rubin, and Bert R. Brown, New York, Academic Press \\ Inc., 1975, 359 pp.
}

\section{René Boulard}

\section{Volume 33, numéro 4, 1978}

URI : https://id.erudit.org/iderudit/028927ar

DOI : https://doi.org/10.7202/028927ar

Aller au sommaire du numéro

Éditeur(s)

Département des relations industrielles de l'Université Laval

ISSN

0034-379X (imprimé)

1703-8138 (numérique)

Découvrir la revue

Citer ce compte rendu

Boulard, R. (1978). Compte rendu de [The Social Psychology of Bargaining and Negotiation, by Jeffrey Z. Rubin, and Bert R. Brown, New York, Academic Press Inc., 1975, 359 pp.] Relations industrielles / Industrial Relations, 33(4), 716-717. https://doi.org/10.7202/028927ar

Tous droits réservés @ Département des relations industrielles de l'Université Laval, 1978
Ce document est protégé par la loi sur le droit d'auteur. L'utilisation des services d’Érudit (y compris la reproduction) est assujettie à sa politique d'utilisation que vous pouvez consulter en ligne.

https://apropos.erudit.org/fr/usagers/politique-dutilisation/ 
L'auteur a ensuite effectué une synthèse ordonnée de ces expressions d'opinion et en a cité plusieurs tout en sauvegardant l'anonymat de ses sources. Les principales conclusions sont condensées dans un résumé de quatre pages et, comme il convient, il n'y a pas de recommandations.

L'étude comprend deux parties divisées en douze chapitres et est suivie d'appendices. La première partie, qui est très brève, situe le problème ainsi que les débats actuels autour des conseils d'administration. C'est la seconde partie qui est la plus importante et qui expose l'auto-critique des directeurs: elle comporte neuf chapitres: le rôle des conseils d'administration, le choix des directeurs et les critères de sélection; l'efficacité des conseils; la situation présente, l'efficacité des conseils: pistes de changements; la responsabilité des conseils; la responsabilité légale des directeurs; les conseils d'administration des filiales de sociétés étrangères; les conseils d'administration des banques; les conseils d'administration des sociétés d'État.

On peut dire qu'à travers ces chapitres, à peu près toutes les questions qui intriguent ceux qui n'ont pas accès à ces chapelles closes, que sont les conseils d'administration, ont été soulevées. Soulignons-en quelques-unes: quels intérêts représentent ces conseils? Qui choisit les membres? A partir de quels critères? Que fait-on des conflits d'intérêt? $\mathrm{Y}$ a-t-il discrimination vis-à-vis des femmes, des Canadiens français? Doit-il y avoir une représentation régionale? Est-ce que l'on s'occupe des actionnaires? Quel pouvoir ont ceux-ci? Doitil y avoir un équilibre entre des directeurs internes et externes?

On note que, dans l'ensemble, les observations fournies dénotent un esprit critique et un dynamisme surprenants. De même aussi, un souci d'adaptation et une acceptation des changements pour s'ajuster aux nouvelles conditions.

À l'heure où un peu partout on parle de réforme de l'entreprise et de démocratie industrielle, il importe de connaître comment fonctionnent ces organismes où se prennent les décisions qui affectent non seulement les sociétés elles-mêmes, mais l'économie du pays. Il est utile aussi de savoir comment se comportent et comment réagissent ceux qui sont aujourd'hui en place.

Cet ouvrage s'inscrit dans le cadre d'une série d'études effectuées par le Conference Board du Canada. Déjà deux études statistiques ont paru: Canadian Directorship Practices : Compensation 1976 et Canadian Directorship Practices: $A$ Profile.

Gérard Dion

Université Laval

The Social Psychology of Bargaining and Negotiation, by Jeffrey $Z$. Rubin, and Bert R. Brown, New York, Academic Press Inc., 1975, 359 pp.

L'ouvrage de Rubin et Brown se présente d'abord comme une monographie ou une revue de la littérature des 15 dernières années sur la psychologie de la négociation. «This book represents perhaps the first attempt to present an extensive overview and analysis of the research in this area " (p. VIII). Ayant recensé 1000 références dans 40 revues différentes, de 1960 à 1974, les auteurs en arrivent à une analyse minutieuse, systématique et détaillée du processus de négociation dans toutes ses dimensions psycho-sociales.

Après avoir clairement défini, à l'aide d'exemples simples, la variable dépendante (la relation de négociation), le volume présente une vue d'ensemble des différentes expériences entreprises pour l'étude de la négociation. Les chapitres 4 à 9 sont consacrés à la description et à l'analyse des différentes dimensions prises comme variables indépendantes, à savoir les facteurs qui affectent l'efficacité de la négociation. Les composantes étudiées sont multiples: chapitre 4: aspect social (présence d'un auditoire, impact d'un tiers, etc.); chapitre 5: aspect physique (lieu, aménagement des locaux, effets d'un échéancier, etc.); chapitre 6: les résultats tangibles et intangibles (v.g. «face saver») de la négociation; chapitre 7: l'influence des caractéristiques de la personnalité des négociateurs; chapitre 8: les orientations interpersonnelles et 
le processus d'interaction; chapitre 9: l'influence sociale et les stratégies de négociation. La lecture est facile, les auteurs évitent les modèles ou théories sophistiqués pour décrire des situations simples (échange d'autos usagées, vacances possibles), avec un vocabulaire simple (facilement accessible à des non-psychologues), mais en poussant la réflexion le plus loin possible, soit pour découvrir des liens entre les résultats de certaines recherches et ainsi les ré-interpréter les unes par rapport aux autres, soit pour tenter d'y découvrir des variables explicatives oubliées des auteurs.

Les auteurs, à l'aide d'un cadre de référence, procèdent à une «re-lecture» des expériences de laboratoire qui ont tenté de contrôler, une à une, les variables liées à la négociation. Il s'agit ici de la grandeur et de la faiblesse du volume. Grandeur puisque l'approche expérimentale permet un contrôle strict des variables: l'approche est systématique; les "évidences» du praticien de la négociation collective reçoivent un appui expérimental, la logique est inattaquable, etc. Surtout, l'approche en laboratoire permet de «démonter»le mécanisme et d'en examiner à la loupe les différents éléments pour mieux comprendre ce qui se passe dans la réalité (v.g. de quelle façon la présence d'un tiers affecte-t-elle le comportement des négociateurs? Quels mécanismes entrent en jeu lorsqu'une échéance stricte est imposée pour en arriver à une entente? Quel est l'impact d'une personnalité autoritaire ou machiavélique sus l'issue de la négociation? etc.). Le souci d'exhaustivité et la préoccupation d'établir chaque proposition sur des données expérimentales ne font jamais défaut et constituent l'apport majeur de cet ouvrage.

Par ailleurs, l'approche expérimentale présente deux inconvénients (que les auteurs reconnaissent volontiers): d'une part, les expériences de laboratoire ne peuvent prétendre au même degré de complexité que la «vraie vie» (v.g. deux étudiants jouant au Monopoly dans un bureau après avoir reçu des directives strictes sur la façon de négocier la valeur des terrains sont loin de la situation des négociateurs à la table centrale dans le secteur public!). D'autre part, les expériences recensées par les auteurs ne cadrent pas toujours avec leur schème de référence ou leur proposition. Ce qui les oblige souvent à «lire entre les lignes» .

L'avantage majeur du volume est de présenter justement un cadre de référence intéressant. Ce qui permet d'une part, de situer la multitude d'expériences examinées, et d'autre part, d'identifier les vides à combler avant de pouvoir élaborer une véritable théorie de la négociation. Le dernier chapitre, après avoir identifié six éléments que les auteurs considèrent comme fort importants à intégrer pour en arriver à cette théorie de la négociation.

À cause, entre autres, du fait que les ouvrages de cette envergure et de cette qualité ne pullulent pas sur la psychologie de la négociation, le volume de Rubin et Brown mérite amplement d'être lu. Le lecteur pressé qui se soucie peu des appuis expérimentaux qui sous-tendent le modèle des auteurs, pourra au moins lire attentivement les deux derniers chapitres et y trouver un intérêt certain, surtout s'il se retrouve à une table de négociation de temps à autre.

René BOULARD

Université Laval

Le travail de nuit: effets sur la santé et la vie sociale du travailleur, par J. Carpentier et $\mathrm{P}$. Cazamian, Genève, Bureau international du travail, $1977,86 \mathrm{pp}$.

Cet ouvrage se présente comme une excellente synthèse des connaissances acquises par la recherche principalement, sur l'impact du travail de nuit pour l'individu.

L'introduction situe le problème du travail de nuit dans ses principales dimensions: situation actuelle du travail de nuit, santé, emploi, évaluation des coûts et avantages économiques et sociaux, réglementation et négociations. Le premier chapitre porte sur les incidences physiologiques, psychologiques et médicales du travail de nuit. Le deuxième chapitre aborde les incidences sur la vie familiale et sociale du travailleur; enfin, le dernier chapitre aborde les aspects ergonomiques. 